\title{
Comparison of the Dirichlet-Neumann and Optimal Schwarz Method on the Sphere
}

\author{
J. Côté ${ }^{1}{ }^{\star}$, M. J. Gander ${ }^{2}$, L. Laayouni ${ }^{3}$, and S. Loisel ${ }^{4}$
}

1 Recherche en prévision numérique, Meteorological Service of Canada, jean.cote@ec.gc.ca

2 Department of Mathematics and Statistics, McGill University, Montreal, mgander@math.mcgill.ca

3 Department of Mathematics and Statistics, McGill University, Montreal, laayouni@math.mcgill.ca

4 Department of Mathematics and Statistics, McGill University, Montreal, loisel@math.mcgill.ca

Summary. We investigate the performance of domain decomposition methods for solving the Poisson equation on the surface of the sphere. This equation arises in a global weather model as a consequence of an implicit time discretization. We consider two different types of algorithms: the Dirichlet-Neumann algorithm and the optimal Schwarz method. We show that both algorithms applied to a simple two subdomain decomposition of the surface of the sphere converge in two iterations. While the Dirichlet-Neumann algorithm achieves this with local transmission conditions, the optimal Schwarz algorithm needs non-local transmission conditions. This seems to be a disadvantage of the optimal Schwarz method. We then show however that for more than two subdomains or overlapping subdomains, both the optimal Schwarz algorithm and the Dirichlet Neumann algorithm need non-local interface conditions to converge in a finite number of steps. Hence the apparent advantage of DirichletNeumann over optimal Schwarz is only an artifact of the special two subdomain decomposition.

\section{Introduction}

Numerical efficiency is very important when modeling the atmosphere, see Côté et al. [1998]. This is particularly true of operational weather forecasts that must be run in real-time during a given time window, and weather being a global phenomenon, one must use a global model to accurately forecast or analyze data. Furthermore, fast waves, which carry little energy, propagate many times faster than the local wind speed, by a factor three or more

\footnotetext{
* The author was partly supported by the Office of Science (BER), U.S. Department of Energy, Grant No. DE-FG02-01ER63199
} 
depending on the application, and these waves restrict the time-step of explicit Eulerian integration schemes. The restrictions are particularly severe for global finite-difference models, due to the convergence of the meridians at the poles. This motivates the use of an implicit (or semi-implicit) time treatment of the terms that govern the propagation of these oscillations in order to greatly retard their propagation and permit a much larger time-step. This approach results in the need to solve an elliptic problem on the sphere. For a time-implicit scheme to be computationally advantageous, it must be possible to integrate with a sufficiently-large time-step to offset the overhead of solving the elliptic-boundary-value problem. This is often the case, even for non-hydrostatic flows, as discussed in Skamarock et al. [1997].

Meteorological operational centers have recently acquired new high-performance significantly-parallel computers. In order to reap the benefits afforded by those systems, parallel algorithms need to be designed for solving the models used in numerical-weather-prediction and data assimilation systems. This motivates the present study, where the parallel solution based on domain-decomposition methods on the surface of the sphere is analyzed. We investigate two domain decomposition methods in this paper: the DirichletNeumann and the optimal Schwarz method. The Dirichlet-Neumann method is a well studied method on the plane, see for example Bjørstad and Widlund [1986], Bramble et al. [1986], Marini and Quarteroni [1989], and references therein. The choice of the optimal relaxation parameter in the DirichletNeumann method on the plane is also well understood: for the case of two subdomains with special symmetry, it is $\frac{1}{2}$. In more general situations, the parameter of relaxation needs to be in a specific interval to obtain a fast algorithm. The key idea underlying the optimal Schwarz method has been introduced in Hagstrom et al. [1988] in the context of non-linear problems. A new class of Schwarz methods based on this idea was then introduced in Charton et al. [1991] and further analyzed in Nataf and Rogier [1995] and Japhet [1998] for convection diffusion problems. For the case of the Poisson equation, see Gander et al. [2001], where also the terms optimal and optimized Schwarz were introduced. Optimal Schwarz methods have in general non-local transmission conditions at the interfaces between subdomains, and are therefore not as easy to use as classical Schwarz methods. Optimized Schwarz methods use local approximations of the optimal, non-local transmission conditions at the interfaces and are therefore as easy to use as the classical Schwarz method, but have a greatly enhanced performance.

In Section 2, we introduce the Poisson equation on the sphere and the tools of Fourier analysis, on which our results are based. In Section 3, we present the Dirichlet-Neumann algorithm for the Poisson equation on the surface of the sphere with possible overlap. We show that convergence in two iterations can be achieved with an appropriate choice of the relaxation parameter. In the case of two subdomains without overlap, this optimal parameter is a constant, but with overlap, and in the case of three subdomains, convergence in a finite number of steps is only possible with a non-local convolution relaxation pa- 
rameter. In Section 4, we present the optimal Schwarz algorithm for the same configuration. We prove convergence in two iterations for the two subdomain case and in three iterations for the three subdomain case, in both cases with non-local transmission conditions. In Section 5 we illustrate our findings with numerical experiments.

\section{The Poisson Equation on the Sphere}

We consider the solution of the Poisson problem

$$
\mathcal{L} u=\Delta u=f, \quad \text { in } \quad S \subset \mathbb{R}^{3},
$$

where $S$ is the unit sphere centered at the origin. Using spherical coordinates, the equation (1) can be rewritten in the form

$$
\mathcal{L} u=\frac{1}{r^{2}} \frac{\partial}{\partial r}\left(r^{2} \frac{\partial u}{\partial r}\right)+\frac{1}{r^{2} \sin ^{2} \psi} \frac{\partial^{2} u}{\partial \theta^{2}}+\frac{1}{r^{2} \sin \psi} \frac{\partial}{\partial \psi}\left(\sin \psi \frac{\partial u}{\partial \psi}\right)=f,
$$

where $\psi$ stands for the colatitude, with 0 being the north pole and $\pi$ being the south pole, and $\theta$ is the longitude. For our case on the surface of the unit sphere, we consider solutions independent of $r$, which simplifies (2) to

$$
\mathcal{L} u=\frac{1}{\sin ^{2} \psi} \frac{\partial^{2} u}{\partial \theta^{2}}+\frac{1}{\sin \psi} \frac{\partial}{\partial \psi}\left(\sin \psi \frac{\partial u}{\partial \psi}\right)=f .
$$

Our results are based on Fourier analysis. Because $u$ is periodic in $\theta$, it can be expanded in a Fourier series,

$$
u(\psi, \theta)=\sum_{m=-\infty}^{\infty} \hat{u}(\psi, m) e^{i m \theta}, \quad \hat{u}(\psi, m)=\frac{1}{2 \pi} \int_{0}^{2 \pi} e^{-i m \theta} u(\psi, \theta) d \theta .
$$

Equation (3) then becomes a family of ordinary differential equations; for any positive or negative integer $m$, we have

$$
-\frac{m^{2}}{\sin ^{2} \psi} \hat{u}(\psi, m)+\frac{1}{\sin \psi} \frac{\partial}{\partial \psi}\left(\sin \psi \frac{\partial \hat{u}(\psi, m)}{\partial \psi}\right)=\hat{f}(\psi, m) .
$$

For $m$ fixed, the homogeneous problem, $\hat{f}(\psi, m)=0$ in (4), has the two fundamental solutions

$$
g_{ \pm}(\psi, m)=\left(\frac{\sin (\psi)}{\cos (\psi)+1}\right)^{ \pm|m|}
$$

Remark 1. $g_{+}$has a singularity at the south pole and $g_{-}$has a singularity at the north pole.

Remark 2. The function $\sin x /(\cos x+1)$ is monotonically increasing on the interval $[0, \pi)$, which can be seen by taking a derivative. 


\section{The Dirichlet-Neumann Algorithm}

We first decompose the surface of the sphere into two overlapping subdomains as shown in Figure 1-(i), where $a \leq b$. The Dirichlet-Neumann method to
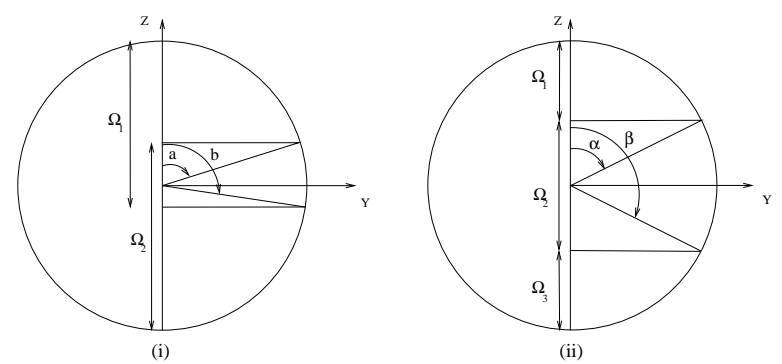

Fig. 1. (i) Two overlapping subdomains (ii) Three nonoverlapping subdomains.

solve (1) solves iteratively the Poisson equation on $\Omega_{1}$ and $\Omega_{2}$ and exchanges Dirichlet and Neumann conditions respectively on the interfaces $a$ and $b$. In its classical form, the algorithm is defined without overlap, but here we are also interested in the influence of overlap on the algorithm. By linearity, it suffices to consider only the homogeneous case in the convergence analysis, $f=0$. For an initial guess $\lambda^{0}(\theta)$ on the interface $b$, the algorithm then performs the iteration

$$
\begin{aligned}
& \mathcal{L} u_{1}^{k+1}=0 \quad \text { in } \Omega_{1}, \quad u_{1}^{k+1}(b, \theta)=\lambda^{k}(\theta), \\
& \mathcal{L} u_{2}^{k+1}=0 \text { in } \Omega_{2}, \quad \frac{\partial}{\partial \psi} u_{2}^{k+1}(a, \theta)=\frac{\partial}{\partial \psi} u_{1}^{k+1}(a, \theta) \text {, }
\end{aligned}
$$

where the new function $\lambda^{k+1}(\theta)$ is defined by the linear combination

$$
\lambda^{k+1}(\theta):=\gamma u_{2}^{k+1}(b, \theta)+(1-\gamma) \lambda^{k}(\theta) .
$$

Here $\gamma$ is a relaxation parameter which is assumed to be non-negative. Expanding the iterates in a Fourier series and taking into account Remark 1 for bounded solutions on the subdomains, we obtain the subdomain solutions

$$
\begin{aligned}
& \hat{u}_{1}^{k+1}(\psi, m)=\hat{\lambda}^{k}(m) \frac{g_{+}(\psi, m)}{g_{+}(b, m)}, \\
& \hat{u}_{2}^{k+1}(\psi, m)=-\hat{\lambda}^{k}(m) \frac{g_{+}(a, m)}{g_{+}(b, m) g_{-}(a, m)} g_{-}(\psi, m) .
\end{aligned}
$$

Letting $\sigma(m)=g_{+}(a, m) g_{-}(b, m)$, the iteration on $\lambda$ in $(7)$ becomes

$$
\hat{\lambda}^{k+1}(m)=\left(1-\hat{\gamma}\left(1+\sigma^{2}(m)\right)\right) \hat{\lambda}^{k}(m) .
$$

Proposition 1. The Dirichlet-Neumann iteration (6) with the two overlapping subdomains on the surface of the sphere converges in two iterations, provided the relaxation parameter is

$$
\hat{\gamma}=\hat{\gamma}_{o p t}(m):=\frac{1}{1+\sigma^{2}(m)} .
$$


Remark 3. The optimal relaxation parameter depends on $m$, which implies a non-local convolution operation in real space. Without overlap however, $a=b$, we have $\sigma(m)=1$, and the optimal relaxation parameter becomes $\gamma_{o p t}=1 / 2$, which is now independent of $m$ and thus a simple local operation in real space.

To see if the optimal result for the non-overlapping two subdomain case can be generalized to more subdomains, we consider a decomposition of the surface of the sphere into three non-overlapping subdomains, see Figure 1-(ii), where $\alpha<\beta$. In this case the Dirichlet-Neumann algorithm for (1) with initial guesses $\lambda_{1}^{0}(\theta)$ and $\lambda_{2}^{0}(\theta)$ is

$$
\begin{aligned}
\mathcal{L} u_{1}^{k+1} & =0 \text { in } \Omega_{1}, & u_{1}^{k+1}(\alpha, \theta) & =\lambda_{1}^{k}(\theta), \\
\mathcal{L} u_{2}^{k+1}=0 & \text { in } \Omega_{2}, & \frac{\partial}{\partial \psi} u_{2}^{k+1}(\alpha, \theta) & =\frac{\partial}{\partial \psi} u_{1}^{k+1}(\alpha, \theta), \\
& & u_{2}^{k+1}(\beta, \theta) & =\lambda_{2}^{k}(\theta), \\
\mathcal{L} u_{3}^{k+1}=0 & \text { in } \Omega_{3}, & \frac{\partial}{\partial \psi} u_{3}^{k+1}(\beta, \theta) & =\frac{\partial}{\partial \psi} u_{2}^{k+1}(\beta, \theta),
\end{aligned}
$$

where the new functions $\lambda_{j}^{k}(\theta), j=1,2$, are defined by

$$
\begin{aligned}
& \lambda_{1}^{k+1}(\theta)=\gamma_{1} u_{2}^{k+1}(\alpha, \theta)+\left(1-\gamma_{1}\right) \lambda_{1}^{k}(\theta), \\
& \lambda_{2}^{k+1}(\theta)=\gamma_{2} u_{3}^{k+1}(\beta, \theta)+\left(1-\gamma_{2}\right) \lambda_{2}^{k}(\theta),
\end{aligned}
$$

and $\gamma_{1}$ and $\gamma_{2}$ are non-negative relaxation parameters. Using Fourier series as before, we arrive at the matrix iteration

$$
\left[\begin{array}{l}
\hat{\lambda}_{1}^{k+1} \\
\hat{\lambda}_{2}^{k+1}
\end{array}\right]=A\left[\begin{array}{l}
\hat{\lambda}_{1}^{k} \\
\hat{\lambda}_{2}^{k}
\end{array}\right], \quad A:=\left[\begin{array}{cc}
\hat{\gamma}_{1} \frac{\Delta_{-}}{\Delta_{+}}+\left(1-\hat{\gamma}_{1}\right) & 2 \frac{\hat{\gamma}_{1}}{\Delta_{+}} \\
-2 \frac{\hat{\gamma}_{2}}{\Delta_{+}} & \hat{\gamma}_{2} \frac{\Delta_{-}}{\Delta_{+}}+\left(1-\hat{\gamma}_{2}\right)
\end{array}\right],
$$

where $\Delta_{ \pm}:=\eta(m) \pm \eta^{-1}(m)$ and $\eta(m):=g_{+}(\alpha, m) / g_{+}(\beta, m)$.

Proposition 2. The Dirichlet-Neumann iteration with three non-overlapping subdomains on the surface of the sphere converges in three iterations, provided the relaxation parameters are

$$
\begin{aligned}
& \hat{\gamma}_{1}=\hat{\gamma}_{1, \text { opt }}(m):=\frac{1}{2}+\frac{1}{2} \eta^{2}(m)-\frac{1}{2} \eta(m) \sqrt{1+\eta^{2}(m)} \\
& \hat{\gamma}_{2}=\hat{\gamma}_{2, \text { opt }}(m):=\frac{1}{2}+\frac{1}{2} \eta^{2}(m)+\frac{1}{2} \eta(m) \sqrt{1+\eta^{2}(m)}
\end{aligned}
$$

This result shows that for more than two subdomains, convergence in a finite number of steps can only be achieved with non-local convolution relaxation parameters in the Dirichlet-Neumann algorithm.

\section{Schwarz Algorithms}

We decompose the surface of the sphere into two overlapping domains as shown in Figure (1)-(i). The classical Schwarz algorithm is given by 


$$
\begin{array}{ll}
\mathcal{L} u_{1}^{k+1}=0 \quad \text { in } \Omega_{1}, & u_{1}^{k+1}(b, \theta)=u_{2}^{k}(b, \theta), \\
\mathcal{L} u_{2}^{k+1}=0, \text { in } \Omega_{2}, & u_{2}^{k+1}(a, \theta)=u_{1}^{k+1}(a, \theta) .
\end{array}
$$

Using a Fourier series expansion as before, we find

$$
\begin{aligned}
& \hat{u}_{1}^{k+1}(a, m)=\rho \hat{u}_{1}^{k}(a, m) \\
& \hat{u}_{2}^{k+1}(b, m)=\rho \hat{u}_{2}^{k}(b, m)
\end{aligned}, \quad \rho:=\frac{g_{+}(a, m)}{g_{+}(b, m)} \frac{g_{-}(b, m)}{g_{-}(a, m)} .
$$

Because of Remark 2, the fractions are less than one and this process is a contraction and hence convergent. We have proved the following

Proposition 3. For each $m \neq 0$, the Schwarz iteration on the surface of the sphere partitioned along two colatitudes $a<b$ converges linearly with the convergence factor

$$
\left(\frac{\sin (a)}{\cos (a)+1}\right)^{2|m|}\left(\frac{\sin (b)}{\cos (b)+1}\right)^{-2|m|}<1 .
$$

This shows that for small values of $m$ the speed of convergence is very poor, since the convergence factor in (17) is nearly one. Following the approach in Gander et al. [2001], we introduce the following new transmission conditions:

$$
\begin{aligned}
& \left(1+\hat{p}(m) \frac{\partial}{\partial \psi}\right) \hat{u}_{1}^{k+1}(b, m)=\left(1+\hat{p}(m) \frac{\partial}{\partial \psi}\right) \hat{u}_{2}^{k}(b, m), \\
& \left(1+\hat{q}(m) \frac{\partial}{\partial \psi}\right) \hat{u}_{2}^{k+1}(a, m)=\left(1+\hat{q}(m) \frac{\partial}{\partial \psi}\right) \hat{u}_{1}^{k+1}(a, m),
\end{aligned}
$$

where $\hat{p}$ and $\hat{q}$ are functions we can use to optimize the performance.

Proposition 4. If, for each $m \neq 0, \hat{p}(m)=\sin (b) /|m|$ and $\hat{q}(m)=-\sin (a) /|m|$, then the new Schwarz algorithm with transmission conditions (18) converges in two iterations, even without overlap, $a=b$.

Proof. Using $\hat{u}_{2}^{1}(\psi, m)=C_{2} g_{-}(\psi, m)$ and $\hat{u}_{1}^{1}(\psi, m)=C_{1} g_{+}(\psi, m)$, where $C_{2}$ is a coefficient to be determined by the method and $C_{1}$ is given through the initial guess, and substituting into the transmission condition, yields

$$
C_{2} g_{-}(a, m)\left(1-\hat{q}(m) \frac{|m|}{\sin (a)}\right)=C_{1} g_{+}(a, m)\left(1+\hat{q}(m) \frac{|m|}{\sin (a)}\right)=0 .
$$

Hence $C_{2}=0$ and the iteration has converged for $\Omega_{2}$. A similar argument shows that in the second step, the iteration converges on $\Omega_{1}$ as well.

To see if this result generalizes to more than two subdomains, we consider the Schwarz algorithm with three subdomains,

$$
\begin{array}{ll}
\mathcal{L} u_{1}^{k+1}=0 \text { in } \Omega_{1}, & \left(1+p \frac{\partial}{\partial \psi}\right) u_{1}^{k+1}(\alpha, \theta)=\left(1+p \frac{\partial}{\partial \psi}\right) u_{2}^{k}(\alpha, \theta), \\
\mathcal{L} u_{2}^{k+1}=0 \text { in } \Omega_{2}, & \left(1+q_{1} \frac{\partial}{\partial \psi}\right) u_{2}^{k+1}(\alpha, \theta)=\left(1+q_{1} \frac{\partial}{\partial \psi}\right) u_{1}^{k+1}(\alpha, \theta), \\
& \left(1+q_{2} \frac{\partial}{\partial \psi}\right) u_{2}^{k+1}(\beta, \theta)=\left(1+q_{2} \frac{\partial}{\partial \psi}\right) u_{3}^{k}(\beta, \theta), \\
\mathcal{L} u_{3}^{k+1}=0 \text { in } \Omega_{3}, & \left(1+r \frac{\partial}{\partial \psi}\right) u_{3}^{k+1}(\beta, \theta)=\left(1+r \frac{\partial}{\partial \psi}\right) u_{2}^{k+1}(\beta, \theta),
\end{array}
$$

where $p, q_{1}, q_{2}$ and $r$ are convolution operators in $\theta$ with Fourier symbol $\hat{p}$, $\hat{q}_{1}, \hat{q}_{2}$ and $\hat{r}$ respectively. 
Proposition 5. If, for each $m \neq 0, \hat{p}(m)=\sin (\alpha) /|m|, \hat{q}_{1}(m)=-\sin (\alpha) /|m|$, $\hat{q}_{2}(m)=\sin (\beta) /|m|, \hat{r}(m)=-\sin (\beta) /|m|$, then the new Schwarz algorithm for three subdomains (19) converges in three iterations.

The proof of this last result is similar to the proof for the two subdomain case.

Remark 4. The choice $\hat{p}(m)=\sin (\alpha) /|m|$ and $\hat{q}_{2}(m)=\sin (\beta) /|m|$ is not necessary in this Gauss-Seidel form of the optimal Schwarz method: $\hat{p}(m)$ and $\hat{q}_{2}(m)$ can be any real number, except $-\sin (\alpha) /|m|$ and $-\sin (\beta) /|m|$ respectively, and the stated results still hold (the situation is similar for the two subdomain case). In the more parallel Jacobi form of the algorithms however the given choice is necessary to obtain the convergence results stated.

\section{Numerical experiments}

We used a spectral method in the longitude with 20 modes, and a finite difference method in the colatitude with discretization parameter $h=\pi / 3000$. In the first set of experiments, we used two subdomains, once with overlap $\left[\frac{9}{20} \pi, \frac{11}{20} \pi\right]$, and once without overlap. A comparison of the convergence behavior of the algorithms is shown in Figure 2 on the left. While the classical
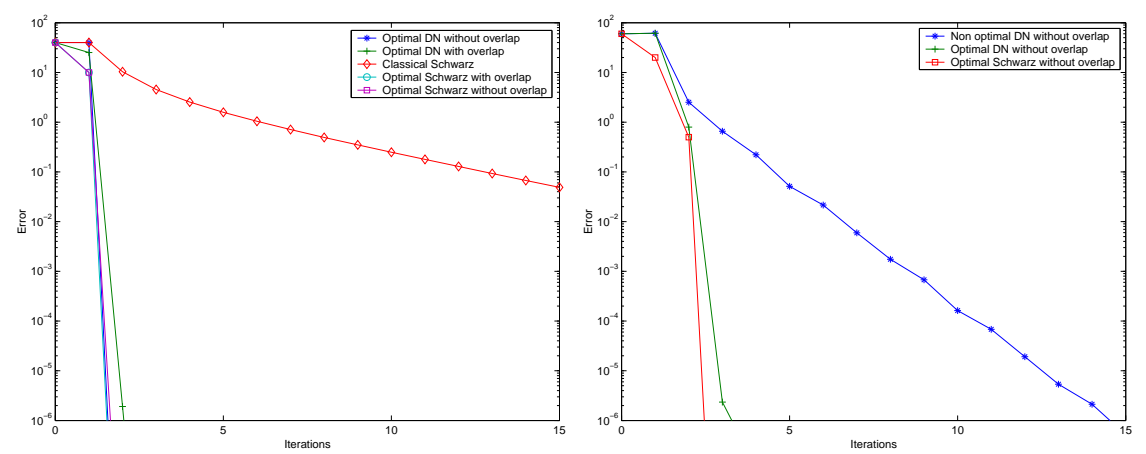

Fig. 2. Convergence behavior for the methods analyzed: the two subdomain case on the left and the three subdomain case on the right.

Schwarz algorithm converges very slowly, both the optimal Schwarz and the Dirichlet-Neumann algorithm converge in two steps with and without overlap, as predicted by the analysis.

In the second set of experiments, we use three non-overlapping subdomains. In Figure 2 on the right, one can see that the optimal Schwarz and Dirichlet-Neumann algorithms converge in three steps, as predicted by the analysis, whereas the Dirichlet-Neumann algorithm with the constant relaxation parameter $1 / 2$, which was optimal for the two subdomain case without overlap, is now much slower. 


\section{Conclusion}

The Dirichlet-Neumann algorithm converges with local relaxation parameter in a finite number of steps only in the special case of two subdomains. To obtain convergence in a finite number of steps for more than two subdomains or if overlap is used, non-local relaxation parameters are needed, like for the optimal Schwarz method. These non-local transmission conditions will serve as a guiding principle to develop local approximations which lead to fast algorithms.

\section{References}

P. E. Bjørstad and O. B. Widlund. Iterative methods for the solution of elliptic problems on regions partitioned into substructures. SIAM J. Numer. Anal., 23(6):1093-1120, 1986.

J. H. Bramble, J. E. Pasciak, and A. H. Schatz. An iterative method for elliptic problems on regions partitioned into substructures. Math. Comp., 46(173):361-369, 1986.

P. Charton, F. Nataf, and F. Rogier. Méthode de décomposition de domaine pour l'équation d'advection-diffusion. C. R. Acad. Sci., 313(9):623-626, 1991.

J. Côté, S. Gravel, A. Méthot, A. Patoine, M. Roch, and A. Staniforth. The operational CMC-MRB global environmental multiscale (GEM) model: Part I - design considerations and formulation. Mon. Wea. Rev., 126:1373-1395, 1998.

M. J. Gander, L. Halpern, and F. Nataf. Optimized Schwarz methods. In T. Chan, T. Kako, H. Kawarada, and O. Pironneau, editors, Twelfth International Conference on Domain Decomposition Methods, Chiba, Japan, pages 15-28, Bergen, 2001. Domain Decomposition Press.

T. Hagstrom, R. P. Tewarson, and A. Jazcilevich. Numerical experiments on a domain decomposition algorithm for nonlinear elliptic boundary value problems. Appl. Math. Lett., 1(3), 1988.

C. Japhet. Optimized Krylov-Ventcell method. Application to convectiondiffusion problems. In P. E. Bjørstad, M. S. Espedal, and D. E. Keyes, editors, Proceedings of the 9th international conference on domain decomposition methods, pages 382-389. ddm.org, 1998.

L. D. Marini and A. Quarteroni. A relaxation procedure for domain decomposition methods using finite elements. Numer. Math, (5):575-598, 1989.

F. Nataf and F. Rogier. Factorization of the convection-diffusion operator and the Schwarz algorithm. $M^{3} A S, 5(1): 67-93,1995$.

W. Skamarock, P. Smolarkiewicz, and J. Klemp. Preconditioned conjugateresidual solvers for Helmholtz equations in nonhydrostatic models. Mon. Wea. Rev., 125:587-599, 1997. 